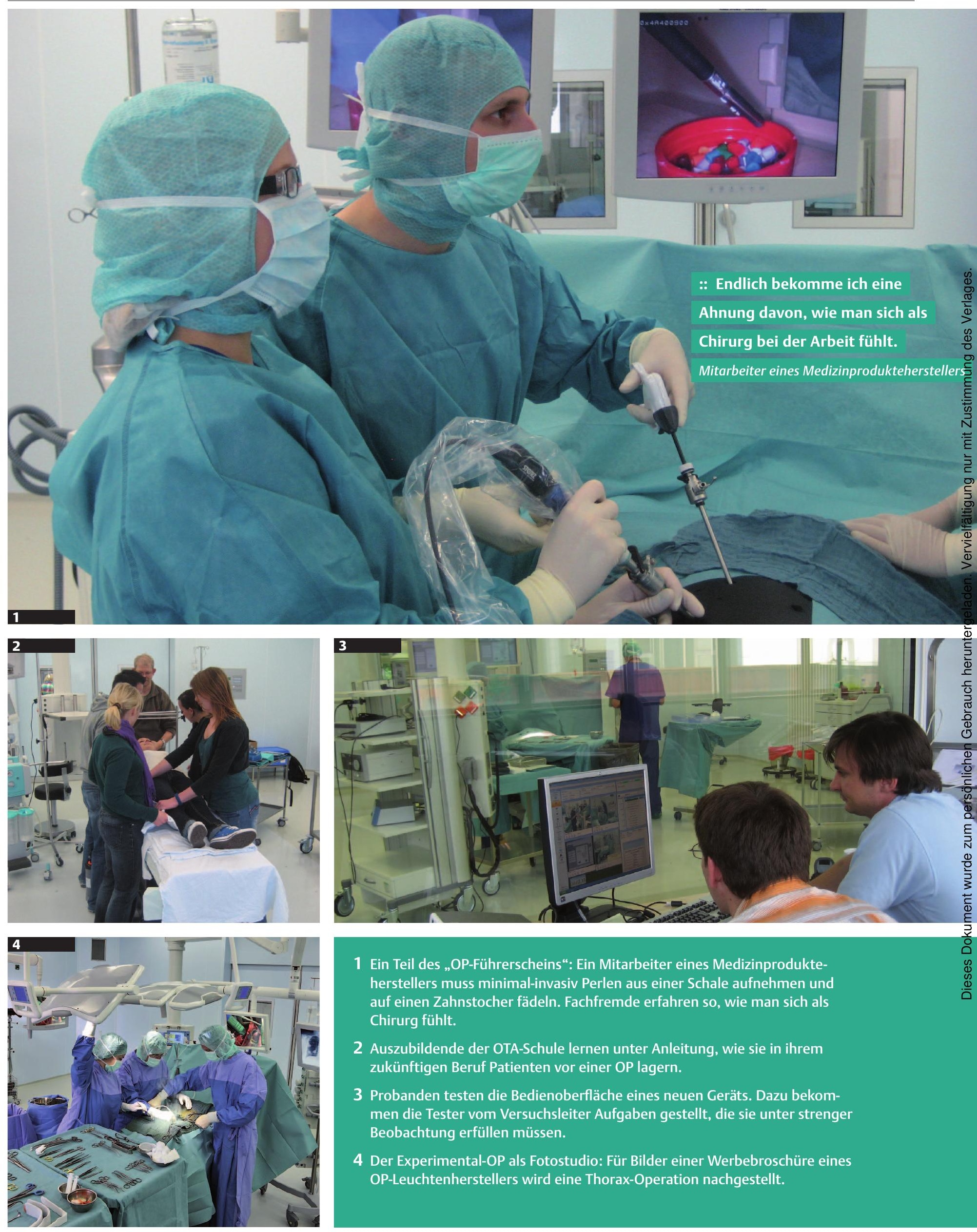

www.thieme.de 


\section{Im Test: Der ideale Arbeitsplatz}

\section{ERSTER EXPERIMENTAL-OP DER WELT IN TÜBINGEN Der Markt für Medizintechnik bietet zahlreiche High-} tech-Geräte für den OP an, mit deren Hilfe Pflegepersonal und Chirurgen vielversprechende Ergebnisse liefern sollen. Aber wie groß ist der Nutzen der Technik, wenn ihre Bedienung ein Ingenieursstudium voraussetzt? Die Gebrauchstauglichkeit neuer Technik ist ein großes Thema. Im ersten Experimental-OP der Welt haben OP-Fachpfleger und Operateure die Möglichkeit, im Vorfeld zu testen und zu bewerten, was morgen auf den Markt kommt.

Hatten Sie schon einmal Probleme damit, ein Gerät im OP korrekt zu bedienen? Oder haben Sie sich über eine unpraktische, umständliche Handhabung geärgert? Dann sind Sie nicht allein. Vor einigen Jahren hat die Studie „Arbeitsbedingungen und Sicherheit am Arbeitsplatz OP“1 gezeigt, dass 50 Prozent der Pflegekräfte und 70 Prozent der Chirurgen nach eigener Aussage Schwierigkeiten haben, die Geräte an ihrem Arbeitsplatz richtig zu bedienen. Wenn aber Mensch und Maschine nicht richtig zusammenfinden, kann es gefährlich werden. Sei es, weil die Geräte einfach zu kompliziert sind oder das Personal nicht richtig eingewiesen ist. Die dabei entstehenden Komplikationen gefährden den Patienten und verursachen hohe zusätzliche Kosten. Wahrscheinlich werden jedes Jahr Hunderte Millionen Euro für die Folgebehandlungen solcher Komplikationen ausgegeben. Im schlimmsten Fall aber führen solche Fehler zum Tod des Patienten.

Autor der genannten Studie ist PD Dr. med. Ulrich Matern. Er hatte als Chirurg selbst oft Probleme mit Instrumenten und Geräten im OP. Zum Beispiel störten ihn die Retraktoren: Das Halten der Instrumente und das Arbeiten mit ihnen bescherte ihm und vielen anderen Operateuren Druckstellen und Parästhesien an den Händen, was nicht nur die eigene Gesundheit gefährdet, sondern auch die des Patienten auf dem OP-Tisch.

\section{Die Idee: Die Arbeit im OP verbessern}

In einer Freiburger Klinik lernte Matern den Krankenhaushygieniker und Ingenieur Martin Scherrer kennen, der, genau wie er selbst, mit einigen Zuständen in der Klinik unzufrieden war. „Wir haben festgestellt, dass es nicht nur mit den Instrumenten Schwierigkeiten gibt, sondern auch Probleme beim Bedienen von Geräten“, erinnert sich Matern. Scherrer störte vor allem die übliche Verdrängungslüftung im OP, die seiner Meinung nach nicht ideal ist: „Bei Standard-Lüftungen wird keimarme Luft über dem OP-Tisch von oben in den Raum geleitet. Das soll gewährleisten, dass die Keimbelastung aus der Luft für den Patienten möglichst gering ist. Faktoren wie Bewegungen des Personals oder die Thermik der Lampe erzeugen aber Turbulenzen, so dass ich nicht an die Funktionalität dieses Systems glaubte." Beide waren sich einig, dass Gebäudetechnik und Medizintechnik in ihrem Haus nicht zusammenpassten, und dass man dagegen etwas tun muss.

Matern und Scherrer hatten eine Idee: Sie wollten ein Labor einrichten, in dem Instrumente, Maschinen, Textilien und alles, was in einen OP gehört, im Vorfeld auf Herz und Nieren von den
Anwendern selbst geprüft wird. „Es reicht nicht, wenn die Sekretärin eines Instrumenteentwicklers sagt: ,Ja Chef, das liegt gut in der Hand'. Es sind OP-Pfleger und Chirurgen, die ihr Arbeitsmaterial beurteilen müssen“, betont Matern. Aus der Vision sollte bald Realität werden. Matern und Scherrer schafften es, die Unterstützung der Universität Tübingen und des Lands Baden-Württemberg zu gewinnen. Zahlreiche Hersteller für Medizinprodukte schenkten dem vielversprechenden Labor Equipment im siebenstelligen Eurobereich oder stellten Dauerleihgaben zur Verfügung. Im Jahr 2008 war es soweit: In eine Fabrikhalle am Stadtrand von Tübingen zog der erste Experimental-OP der Welt ein.

Das Herzstück der 1000 Quadratmeter messenden Anlage sind ein großer und ein kleiner OP-Saal, ein Einleitungs- sowie Aufwachraum und ein Krankenzimmer, das zum Beispiel als Intensivzimmer umgebaut werden kann. Außerdem gibt es viel Raum für theoretisches Training, Vorträge und Seminare. Die OP-Säle sind komplett und modern eingerichtet, am Boden liegt kein Kabel, fast alle Geräte sind an der Decke montiert. Hier finden niemals Operationen an Mensch oder Tier statt, sondern ausschließlich an Attrappen. So können Maschinen und Instrumente unter realen Bedingungen getestet oder Schulungen durchgeführt werden, ohne dass jemandem geschadet wird. An den Wänden sind Videokameras und Mikrofone angebracht, die jeden Test zur späteren Auswertung mitschneiden. Zudem gibt es einen kleinen Raum, von dem aus man das Geschehen im großen OP durch eine Glas-

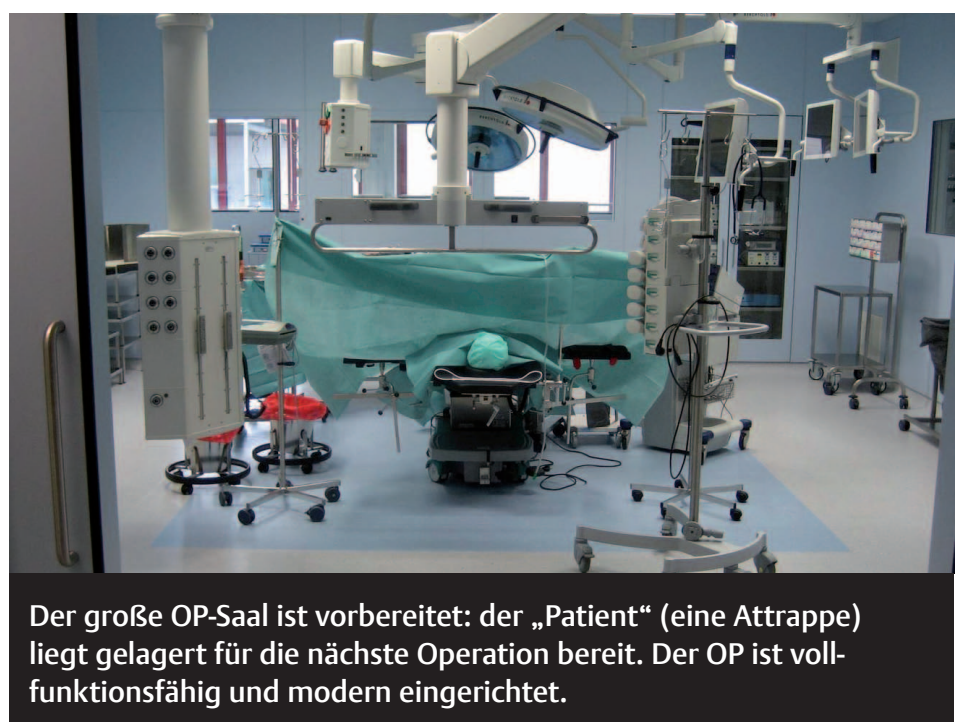


scheibe hindurch beobachten kann. Neben den Türen zu den Operationssälen ist außen ein Desinfektionsmittelspender installiert, mit dem man sich die Hände desinfizieren muss, sonst wird der Zugang zum OP verwehrt. Die Türen selbst sind über zwei Meter breit. Der Vorteil: Patienten können im Einleitungsraum vollständig gelagert und erst dann in den OP geschoben werden, selbst wenn sie zwei Meter groß sind und mit gespreizten Armen oder Beinen operiert werden sollen. Dadurch kann die Zeit im OP effizienter genutzt werden, was auch in der Klinik Kosten sparen würde.

Seit letztem Jahr ist aus dem Experimental-OP die eigenständige Firma wwH-c (worldwide Hospital competence) geworden, für die ein Chirurg, eine OP-Fachpflegerin, zwei Ingenieure und eine Betriebswirtin arbeiten. „Wir sind eine gute Mischung, weil wir unterschiedliches Fachwissen mitbringen und alle etwas gemeinsam haben: Wir haben gelernt, über den Tellerrand hinauszuschauen“, beschreibt Matern das Team. Er leitet zusammen mit Scherrer die neue GmbH, bei der auch Diana Hagen angestellt ist. Sie bringt 18 Jahre Erfahrung als OP-Fachpflegerin mit. Oft steht sie selbst am OP-Tisch und testet die Bedienung von Geräten oder führt Schulungen durch.

\section{Das Problem: Entwickler sind keine Anwender}

Als Hagen noch in der Klinik arbeitete, kamen oft Vertreter von Medizinprodukten mit einem Gerät in die Klinik und baten das OP-Personal, die Gebrauchstauglichkeit zu testen. „Damit das Gerät nicht verloren ging, wurde es eingeschlossen. Wenn der Vertreter einige Wochen später wiederkam, musste er sein Gerät ungetestet wieder mitnehmen, denn im Klinikstress wurde meist vergessen, das Produkt auszuprobieren“, erinnert sie sich.

In die Entwicklung eines medizinischen Gerätes sind üblicherweise mehr Ingenieure eingebunden als OTAs oder Chirurgen, denn der primäre Fokus liegt auf der technischen Funktionalität. Experimental-OP-Leiter Matern wünscht sich aber, dass die Hersteller sich von Anfang an beraten lassen und mit den tatsächlichen Anwendern über Ergonomie sprechen, damit das neue Ge-

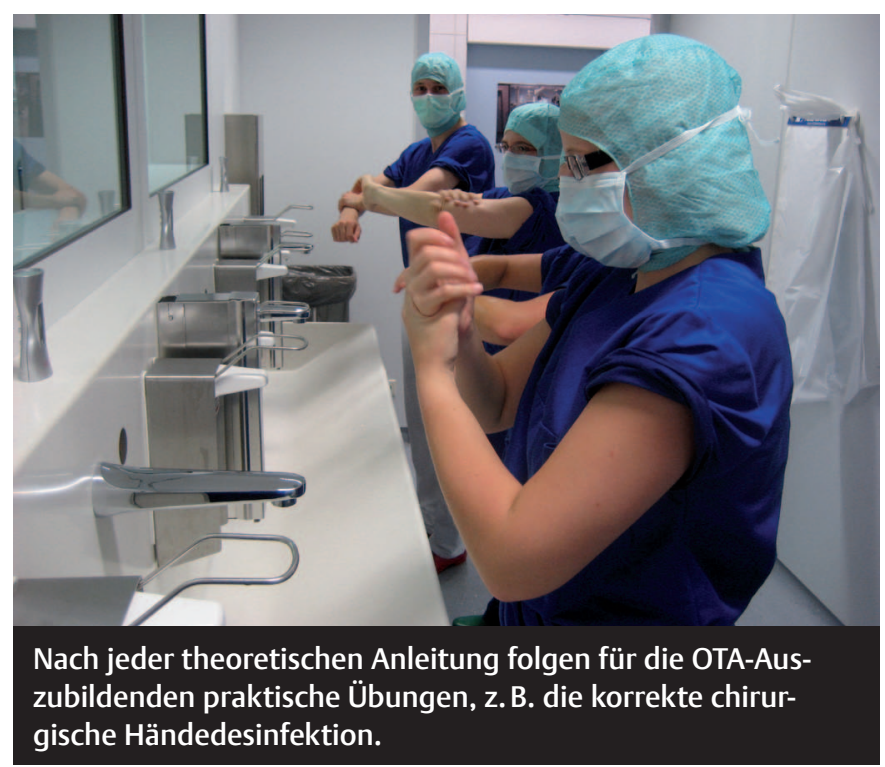

rät in all seinen Funktionen auch bequem nutzbar ist. Folgendes Schlüsselerlebnis zeigte Matern, wie wichtig es ist, dass Ingenieure wissen, wie es im OP zugeht: Eine österreichische Firma, die eigentlich Produkte für die Zahnmedizin herstellt, wollte eine Säge für die Knochenchirurgie produzieren. Für zwei Tage reiste der Chefentwickler mit seinem sechsköpfigen Team nach Tübingen, um dort operieren zu lernen. Matern ernannte ihn zum Operateur einer simulierten Gallen-OP. „Es fing so an, dass der Operateur seine Mitarbeiter höflich und in vollständigen Sätzen bat, dort und hier doch bitte ein bisschen zu ziehen oder ihm ein Instrument zu reichen. Nach zehn Minuten hatte er verstanden, dass es so nicht funktioniert, und warum man im OP nicht diskutiert. Er war so konzentriert auf die Galle, dass er sich wie im realen OP verhielt." Matern hatte sein Ziel erreicht: Die Entwickler fühlten und dachten wie Fachpersonal. Solche Schulungen können das Augenmerk der Entwickler schon während der Entstehung eines neuen Produkts auf dessen Gebrauchstauglichkeit lenken.

\section{Die Lösung: Bedienungsprobleme im Experiment aufdecken}

Die öfter gewählte Möglichkeit besteht darin, die Geräte im Nachhinein von Anwendern testen zu lassen. Leider gibt es dazu kaum gesetzliche Vorschriften. Ein Hersteller von medizinischen Geräten ist nicht verpflichtet, seine Produkte vor der Markteinführung auf Bedienungsfreundlichkeit testen zu lassen. Allerdings gibt es seit 2008 zwei Normen, die dem Hersteller Vorschriften machen: So soll für jedes Gerät vor der Zulassung eine „Ergonomie-Akte“ angelegt werden. Darin sollen auch sogenannte Gebrauchstauglichkeitsstudien enthalten sein - wie diese allerdings auszusehen haben, bleibt offen.

Das Team des Tübinger Experimental-OPs hat dafür einen Standard mit einem genauen Bewertungssystem entwickelt. Personen aus verschiedenen medizinischen Berufen benutzen als Produkttester in simulierten OPs ein Gerät. Dazu bekommen sie von OPFachpflegerin Hagen standardisierte Aufgaben gestellt. Beispielsweise müssen sie mit einem zu erprobenden OP-Mikroskop ein Bild schießen oder eine bestimmte Funktion am Gerät aktivieren.

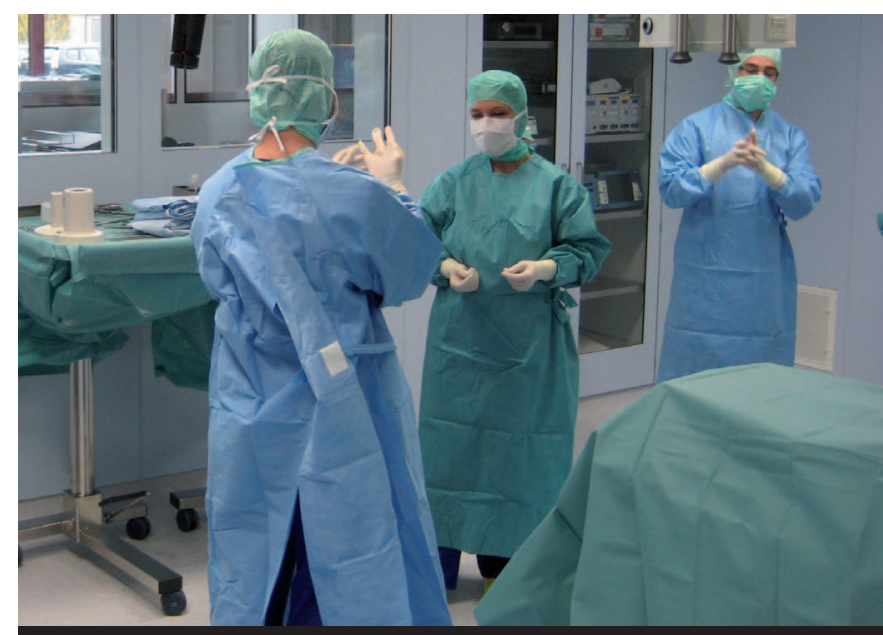

Wer den „OP-Führerschein“ bestehen möchte, muss lernen, wie man sich im OP verhält. Dazu gehört auch die knifflige Aufgabe, sterile Handschuhe richtig anzuziehen. 

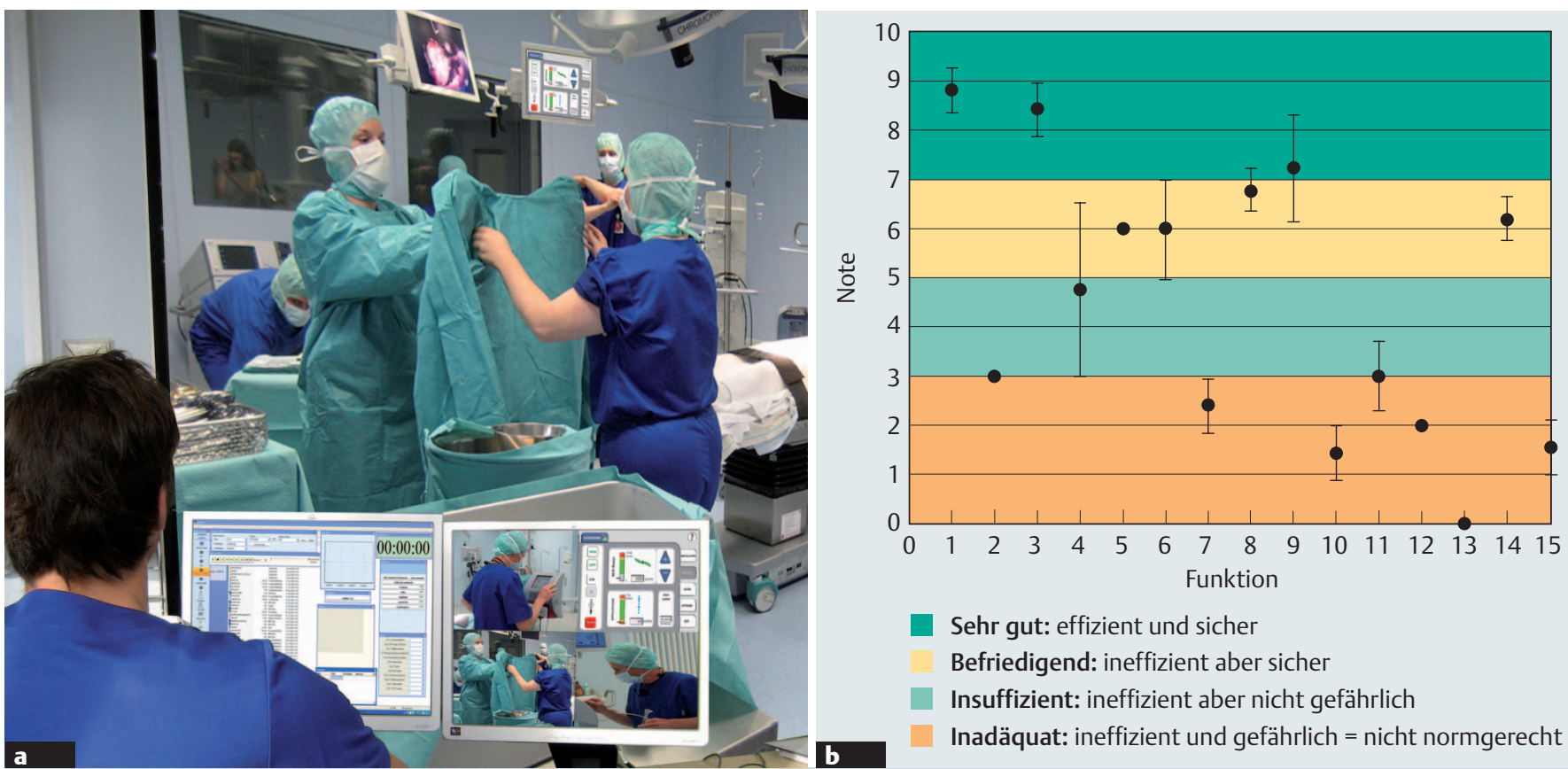

Sehr gut: effizient und sicher

Befriedigend: ineffizient aber sicher

Insuffizient: ineffizient aber nicht gefährlich

Inadäquat: ineffizient und gefährlich = nicht normgerecht

Abb. 1 Beim Gebrauchstauglichkeitstest wird jeder Handgriff von einer Videokamera aufgezeichnet und beobachtet (a). Die Ergebnisse werden in einem „Notenblatt“ (b) festgehalten und dem Hersteller vorgelegt. Die waagerechte Achse bildet die zu testende Funktion des Geräts ab (in diesem Beispiel 15), während die senkrechte Achse die Bewertung von 1 bis 10 angibt.

Nachdem alle Produkttester die Aufgaben ausgeführt haben, werden in einer Expertenrunde die Probleme ausgewertet, die sich bei der Bedienung ergeben haben. Zu den Experten zählen Ingenieure aus den Reihen des Herstellers, externes OP-Personal, das normalerweise in Kliniken arbeitet, und meist Firmenleiter Matern sowie Diana Hagen. Zudem können auch die Testpersonen selbst nach ihren Eindrücken befragt werden.

Der Ablauf einer typischen Auswertungsrunde: Ein Moderator präsentiert die Funktionen des Produktes und die Anwendungsprobleme, die in der Testphase aufgetreten sind. Zum Beispiel könnten 30 Prozent der Testpersonen den Einschaltknopf nicht gefunden haben. Die Runde diskutiert dann, wie wichtig das Problem ist und welche Konsequenzen es in der Realität auslösen würde. Für viele Produkte ist es wenig relevant, ob 30 Prozent der Nutzer das Gerät nicht sofort anschalten können, aber bei einem Defibrillator hätte das schlimme Folgen. Nach der Diskussion vergibt jeder Noten von eins bis zehn für die besprochene Funktion. Noten von eins bis drei bedeuten, dass diese Funktion unsicher und ineffizient ist, weil dabei eine Person gefährdet wird oder der Patient sogar gestorben wäre, die Noten acht bis zehn dagegen stehen für „sehr gute“ Handhabung und Gebrauchstauglichkeit $(\rightarrow$ Abb. 1).

Sämtliche Ergebnisse werden zusammengefasst und in einer Art Zeugnis dem Hersteller vorgelegt. Doch nicht immer lässt sich der Hersteller davon beeindrucken: „Es gibt genügend Geräte, die Funktionen besitzen, die als ineffizient benotet wurden. Sie wurden aber trotzdem unverändert auf den Markt gebracht“, bedauert Matern. Dennoch blickt er optimistisch in die Zukunft: „Eines unserer Ziele ist, dass es das irgendwann nicht mehr geben wird.“

\section{Einfache Anwendung spart Kosten}

Eine der seit 2008 bestehenden Normen verpflichtet Hersteller von medizinischen Geräten, anzugeben, wie groß der Schulungsund Trainingsaufwand für ihr Produkt ist. Diesen Punkt hält Matern für äußerst wichtig. In einer Studie fand sein Team heraus, dass Einweisungen an medizinischen Geräten nur dann etwas bringen, wenn sie als praktisches Training gestaltet werden. Praktische Schulungen kosten aber viel Zeit und Geld, wobei regelmäßige Nachschulungen die Kosten noch in die Höhe treiben. Gut durchdachte Produkte sind hier klar im Vorteil: Sie verschlingen weniger Zeit und Geld für Trainings und sind im Notfall intuitiv einfacher zu bedienen. Matern sieht hierin das größte Potenzial für sein Projekt: „Wenn die Krankenhäuser von dieser Norm wüssten und sie in ihre Entscheidung, ein Gerät anzuschaffen oder nicht, einfließen lassen würden, dann hätten ergonomische Aspekte plötzlich einen viel höheren Stellenwert. Dann würden sich endlich die Produkte durchsetzen, die einfach und sicher zu bedienen sind. Aber bis dahin ist es noch ein langer Weg, weil viele Entscheider in Krankenhäusern die Normen einfach nicht lesen.“

Ein interessantes Gerät, das sich gerade im Test befindet, ist eine neuartige Lüftungsanlage, die fest im großen OP installiert ist. Ihre Besonderheit liegt in der Strömungsrichtung der Luft. Herkömmliche Umluft-Klimaanlagen speisen die Luft von oben in den OP ein und verteilen sie dann. Eine Simulation hat aber gezeigt, dass sich damit der chirurgische Rauch, der bei der diathermischen Erhitzung von Gewebe entsteht, in kürzester Zeit im gesamten OP verteilt. Personal und Patienten werden dabei verschiedensten schädlichen Partikeln und Chemikalien ausgesetzt, darunter auch das Karzinogen Benzol. Das neue System belüftet den Raum von 


\section{DER EXPERIMENTAL-OP AUF EINEN BLICK}

- Eröffnung: 2008

- Bauherr: Universitätsklinikum Tübingen

- Finanzierung: Universitätsklinikum und Medizinische Fakultät Tübingen, Land Baden-Württemberg

- Medizinische Ausstattung: Gespendet von rund 80 Medizinprodukteherstellern (Schenkungen und Dauerleihgaben im Wert von circa drei Millionen Euro)

- Räumlichkeiten: Insgesamt 1000 qm (400qm OP-Trakt, 600 qm Technik-, Konferenz- und Büroräume) in einer ehemaligen Fabrikhalle am Stadtrand von Tübingen

- Ausstattung des OP-Trakts: Zwei voll funktionsfähige OP-Säle, Einleitungs- und Aufwachraum, Patientenzimmer, Sterilgutlager mit -versorgung

- Anzahl der Mitarbeiter: Fünf (ein Chirurg, zwei Ingenieure, eine OP-Fachpflegerin, eine Betriebswirtin) in einer seit 2009 eigenständigen Firma (wwH-c GmbH)

unten und transportiert die Luft nach oben über den OP-Tisch ab. Eine weitere Simulation zeigte, dass der Diathermie-Rauch damit wesentlich besser abzieht. Staub und Keime vom Boden werden bei dieser Methode dabei trotzdem nicht vermehrt hochgeblasen: „Bis jetzt haben alle Messungen ergeben, dass auf dem OP-Tisch keine höhere Bodenstaubbelastung als bei anderen Lüftungssystemen entsteht“, so Matern. Bisher wurde die Anlage nur zur Probe eingesetzt. Bald möchte man das Lüftungssystem aber in einer Studie am Patienten testen. Matern sähe in der Einführung einen großen Fortschritt für den Arbeitsschutz.

\section{Fehler ohne Folgen in der Ausbildung}

Dank des voll funktionsfähigen OP-Trakts können OTAs hier einige praktische Teile ihrer Ausbildung absolvieren. Vom Einschleusen über das Instrumentieren bis zur Lagerung lernen sie ihren zukünftigen Arbeitsplatz kennen und können Dinge selbst ausprobieren, wie beispielsweise verschiedenste Osteosyntheseverfahren an Kunstknochen. OP-Fachpflegerin Hagen schätzt vor allem den Vorteil, dass die Auszubildenden dabei nicht mit Patienten in Kontakt kommen und keine Infektionsgefahr besteht: ,Junge Kollegen sind oft stark damit beschäftigt, alles richtig zu machen und denken weniger darüber nach, ob sie sich mit einer Krankheit infizieren könnten. Bei uns hat es keine Folgen, wenn man nach einem bestimmten Handgriff vergisst, die Hände zu desinfizieren.“ Auch brauchen die Auszubildenden nicht wie in der Klinik darauf zu warten, dass der OP einmal frei ist, was meist abends oder am Wochenende der Fall ist, und selbst dann kommen oft Notfälle dazwischen.

Das Ausbildungsangebot im Experimental-OP ist gefragt, wobei der „OP-Führerschein“ besonders beliebt ist. Alle Medizinstudenten der Uni Tübingen kommen im vierten Semester hierher und lernen in praktischen Übungen, wie man sich rund um den OP verhält und was man auf keinen Fall tun sollte. Als Belohnung für den bestandenen Schein dürfen die Studenten schon im vierten Semester im realen OP assistieren. Die Deutsche Gesellschaft für Chirurgie (DGCH) unterstützt dieses Angebot und verspricht sich davon, dass die Patientensicherheit im realen OP verbessert

\section{WAS PASSIERT IM EXPERIMENTAL-OP?}

Alles rund um den Operationsbereich, von der Kleidung über Arbeitsabläufe bis hin zur Beleuchtung kann hier erforscht, analysiert, getestet und geschult werden. Alle Tests werden ausschließlich an Attrappen durchgeführt. Ohne dass ein Patient oder Tier zu Schaden kommt, wird das Labor vielseitig genutzt in folgenden Bereichen:

- Medizinprodukte: Forschung, Entwicklung, Gebrauchstauglichkeitstests nach internationalen Normen, Verbesserung der Mensch-Maschine-Schnittstelle

- Schulungen: OTA- und ATA-Ausbildung, vierwöchige OP-TeamAssistent-Ausbildung, OP-Führerschein für Medizinstudenten oder Mitarbeiter von Medizinprodukteherstellern, Kurs in der Sterilgutaufbereitung, Schulung über Gebrauchstauglichkeit

- Hygiene: Forschung, Entwicklung und Tauglichkeitstest

- Arbeitsabläufe: Überprüfung auf Effizienz und Sicherheit, Weiterentwicklung

wird. Matern könnte sich sogar vorstellen, dass man in ferner Zukunft ohne einen OP-Führerschein keinen OP mehr betreten darf.

Dieses Jahr im Winter wird außerdem erstmals ein vierwöchiger Lehrgang zum „OP-Team-Assistenten“ (OpTeamA) angeboten. Neue Mitarbeiter, die Hilfstätigkeiten im OP leisten, wissen oft nicht, wie sie sich richtig verhalten sollen. Im stressigen Alltag bleibt wenig Zeit, es ihnen zu erklären - damit stellen sie für das erfahrene OP-Personal manchmal eine Belastung dar. Auch die Neulinge selbst fühlen sich unsicher. Im Experimental-OP kann man in kurzer Zeit grundlegende Dinge wie beispielsweise Hygiene, Strahlenschutz, Grundlagen der Lagerung oder Kommunikation im OP erlernen oder auf den neusten Stand bringen, beispielsweise nach einem längeren Erziehungsurlaub. Die Team-Assistenten dürfen zwar nicht am Tisch stehen und assistieren, aber sie können OP-Fachpflegern einfachere Tätigkeiten abnehmen, um sie zu entlasten. Matern hofft, dass man mit den Team-Assistenten die Personalknappheit in den OPs reduzieren kann. Sie könnten je nach beruflicher Vorbildung (Nicht-Intensiv-) Patienten empfangen und in den OP begleiten oder den OTAs bei Vorbereitungs- und Aufräumarbeiten helfen.

Der Experimental-OP ist in der kurzen Zeit seines Bestehens zu einer vielseitigen Einrichtung geworden, die für Schulungen genutzt wird und gleichzeitig als Testlabor, Konferenzzentrum und Dauerausstellung dient. Und seine Geschichte hat gerade erst begonnen - schließlich gibt es in den Kliniken noch viel zu verbessern. Die Leiter stecken voller Tatendrang: Matern sieht noch eine lange Liste mit Mängeln in Krankenhäusern vor sich, die behoben werden müssen. Scherrer hat noch viele Ideen, die Gebäude- und Medizintechnik zu optimieren und einander anzupassen. Beide denken daran, die Studie „Arbeitsbedingungen und Sicherheit am Arbeitsplatz OP“" aus dem Jahr 2006 irgendwann zu wiederholen. Eine Verbesserung der Ergebnisse wäre die schönste Bestätigung dafür, dass sie auf dem richtigen Weg sind.

\section{Literatur}

1 Matern U et al. Arbeitsbedingungen und Sicherheit am Arbeitsplatz OP. Dtsch Arztebl 2006, 103(47):A3187-92 


\section{Nachgefragt ...}

... BEI MICHAEL WALZ, Textilingenieur bei der Eschler Textil GmbH, Partnerfirma des Experimental-OPs

\section{- Was gefällt Ihnen an Ihrer Zusammenarbeit mit dem Experi- mental-OP?}

Wir stehen immer im direkten Kontakt mit Menschen aus dem OP. Das ist wichtig für uns, denn unsere Firma entwickelt sämtliche Textilien, die man im OP findet: Stoffe für Bekleidung, Patientendecken oder Auflagen, Abdeck- und Containertücher. Normalerweise ist es für Hersteller schwierig, direkt mit Angestellten aus dem OP über ihre Bedürfnisse zu den Textilien - zum Beispiel zum Tragekomfort der Bekleidung - zu sprechen. Im Experimental-OP arbeiten Menschen, die selbst aus der Klinik kommen und viel Zeit im OP verbracht haben. Sie haben viel Erfahrung und wissen, worauf es ankommt. Die Rückmeldungen und Anregungen, die wir von dort aus erster Hand bekommen, sind für uns sehr wertvoll.

\section{- Wie kam es zu der Partnerschaft?}

Bevor der OP gebaut wurde, gab es eine Informationsveranstaltung, die auch dazu diente, Partnerfirmen zu gewinnen. Wir waren sofort begeistert von dem Projekt. Für uns hieß es dann aber erst einmal „Abwarten“, weil zuerst alles aufgebaut werden musste. Schließlich haben wir gemeinsam mit unserer Partnerfirma Dieckhoff die komplette OP-Bekleidung zu Verfügung gestellt. Die Materialbereitstellung hatte ungefähr einen Wert von 4000 Euro, was im Vergleich zu einem OP-Tisch oder anderen Geräten ein eher kleiner Betrag ist.

\section{Was ist das Besondere an der Bekleidung?}

Sie besteht aus Mikrofasern, die bereits seit einiger Zeit im Sportbereich eingesetzt werden. Gegenüber herkömmlicher Bekleidung aus Baumwolle bieten diese Mikrofasern sehr guten Tragekomfort, verbessern die Hygiene im OP und erleichtern die Wiederaufbereitung. Die normalen Körperfunktionen werden laut Bekleidungsphysiologie unterstützt.

\section{- Was heißt das genau?}

Speziell konstruierte Funktionskleidung aus Mikrofaser transportiert den Schweiß nach außen und trocknet schneller. Das verbessert den Tragekomfort und trägt dazu bei, dass Arbeitende ihre Leistungsfähigkeit über mehrere Stunden halten können. Unter dem OP-Mantel ist es sehr warm, weil bei Einwegmaterialien oft wenig Atmungsaktivität herrscht, da merkt man den Unterschied deutlich. Mikrofaser ist auch hygienisch der Baumwolle überlegen, denn Stapelfasergarne aus Baumwolle und Baumwollmischungen begünstigen den Faserflug, der dazu führt, dass Erreger in der Raumluft transportiert werden können. Bei der Wiederaufbereitung benötigt das Mikrofasermaterial weniger Energie, weil es kürzere Trocknungszeiten aufweist. Im Moment wird die Kleidung im Experimental-OP verwendet und in einigen Kliniken getestet.

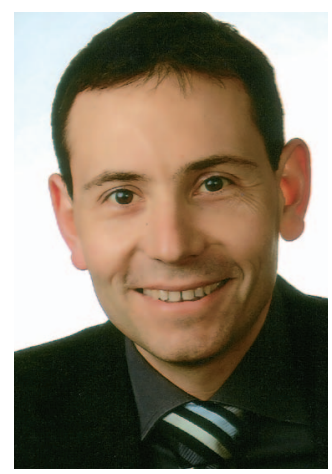

: Kliniken müssen die Hygiene immer wichtiger nehmen. Die Baumwolle wird deshalb verschwinden.

\section{- Wird sich die Mikrofaser irgendwann in den Krankenhäusern durchsetzten?}

Es wird auf jeden Fall eine Veränderung geben, weil die Kliniken die Hygiene immer wichtiger nehmen müssen. Die Baumwolle wird verschwinden, welches Material genau sich durchsetzen wird, ist aber noch nicht abzusehen. In Skandinavien beispielsweise gibt es schon Krankenhäuser, die auf Baumwolle in den Textilien verzichten. Ich hoffe, dass die Umstellung bei uns im nächsten Jahr beginnen wird.

- Welchen Vorteil haben Sie aus dieser Partnerschaft gezogen? Wir haben einen wertvollen Partner zum Erfahrungsaustausch gewonnen. Wir bekamen nicht einfach einen Bericht, der uns bestätigte, wie gut unsere Produkte sind. Die Textilien sind vielmehr eine Investition in die Beziehung zum Experimental-OP. Wir sind im ständigen Austausch über Vor- und Nachteile, die den Probanden auffallen und halten Rücksprache über neue Ideen. Für mich persönlich war meine Teilnahme am OP-Führerschein sehr eindrucksvoll. Ich stand einen halben Tag in voller Montur im OP, was ich vorher nur aus der Theorie kannte. Das hat mich der Praxis einen großen Schritt näher gebracht hat.

\section{AUTORIN \\ Mona Herz \\ Journalistin und Medizinstudentin \\ Freie Autorin des Georg Thieme Verlags seit 2008}

\section{BIBLIOGRAFIE}

DOI 10.1055/s-0031-1271608

ImOP 2011; 2: 52-57

(C) Georg Thieme Verlag KG

Stuttgart . New York . ISSN 1611-7905 